PROCEEDINGS OF THE

AMERICAN MATHEMATICAL SOCIETY

Volume 138, Number 5, May 2010, Pages 1575-1580

S 0002-9939(10)10078-1

Article electronically published on January 19, 2010

\title{
APPROXIMATE MULTIPLICATIVE GROUPS IN NILPOTENT LIE GROUPS
}

\author{
DAVID FISHER, NETS HAWK KATZ, AND IRINE PENG
}

(Communicated by Michael T. Lacey)

\begin{abstract}
We generalize a result of Tao which describes approximate multiplicative groups in the Heisenberg group. We extend it to simply connected nilpotent Lie groups of arbitrary step.
\end{abstract}

\section{INTRODUCTION}

Given a set $A$ in an abelian group, we say it has small additive doubling if

$$
|A+A| \leq K|A|,
$$

where $K$ is a constant which is small in an appropriate sense. The aim of the classical Freiman's theorem is to show that sets having small additive doubling exhibit a lot of structure.

Terry Tao in 4 studied the analogous situation for sets of small multiplicative tripling in a Heisenberg group. He showed that such a set can be mapped into an abelian group in such a way that it has additive structure which is consistent with commutation. Our aim is to obtain such a result in the setting of general simply connected nilpotent Lie groups. Our only tool will be a rather direct application of the Baker-Campbell-Hausdorff formula.

We will investigate the structure of a subset $A \subset N$ which is an approximate multiplicative group. We recall that an approximate multiplicative group is a set $A$ with the property

$$
A A \subset \bigcup_{l=1}^{k} x_{l} A,
$$

where the $x_{l}$ 's are elements of $N$ and we refer to $k$ as the multiplicative constant of $A$. We will restrict ourselves to approximate multiplicative groups which are symmetric: $A=A^{-1}$. The relations between symmetric approximate multiplicative groups and sets with small tripling are discussed in [4] and [3].

Our goal is to prove the following theorem:

Theorem 1.1. Let $A$ be a symmetric approximate multiplicative group in a simply connected, nilpotent, $n$-step Lie group $N$.

Received by the editors January 29, 2009, and, in revised form, June 7, 2009.

2010 Mathematics Subject Classification. Primary 20-XX; Secondary 05-XX.

(C)2010 American Mathematical Society

Reverts to public domain 28 years from publication 
(1) We have that for any small set of small integers $k_{1}, k_{2}, \ldots, k_{l}$ that

$$
\left|\log \left(A^{k_{1}}\right)+\log \left(A^{k_{2}}\right)+\cdots+\log \left(A^{k_{l}}\right)\right| \lesssim|\log (A)|,
$$

with the constants depending only on the multiplicative constant of $A$, the step $n$, the maximum of the $\left|k_{j}\right|$ 's and $l$.

(2) Let $B_{0}=\log (A)$ and $B_{j}$ be $\left[B_{0}, B_{j-1}\right]$. (By nilpotence this sequence terminates.) Then for each $j$, there is a number $l$, integers $k_{1}, \ldots, k_{l}$ and rationals $q_{1}, \ldots, q_{l}$ depending just on $j$ and $n$ so that

$$
B_{j} \subset q_{1} \log \left(A^{k_{1}}\right)+q_{2} \log \left(A^{k_{2}}\right)+\cdots+q_{l} \log \left(A^{k_{l}}\right) .
$$

Morally, this result says that if $A$ is a symmetric approximate multiplicative group, then $\log (A)$ is very close to being a Lie algebra. In particular, the first statement says that the $\log$ of small powers of $A$ all have small additive doubling and are almost additively parallel. The second part says that the iterated commutators of $\log (A)$ (which by nilpotence live in progressively smaller subspaces of the Lie algebra) are also additively compatible with sets from the first part. In particular, a set $A$ having just these two properties automatically has small multiplicative tripling by applying the Baker-Campbell-Hausdorff formula so that we essentially have a characterization of such sets.

Further, one can apply the classical Freiman's theorem to $\log (A)$ and then use the second condition in our theorem to impose further restrictions on the resulting generalized arithmetic progression. It is also fairly easy to adapt our arguments to the setting of torsion nilpotent groups, at least when all elements have an order that is large enough. Here large enough is in terms of various constants arising in the Baker-Campbell-Hausdorff formula. Combining this with the theorem above and known facts about the structure of nilpotent groups gives a result for all nilpotent groups without small torsion.

We mention here the analogue for multiplicative subgroups of $N$ of the key step in our proof; namely, if $G$ is a subgroup of $N$, then there is a subgroup $V$ of $\mathfrak{n}$ and an integer $c>1$ such that $c V \subset \log (G) \subset V$. One can deduce this easily from Lemma 2.2 below. It is also not hard to deduce this fact about groups from classical results of Malcev; see 11 or [2, Chapter 2]. To deduce this fact from the classical results, one needs to first use that since $G$ is torsion free, there is a simply connected nilpotent group $N^{\prime}$ in which $G$ is a lattice. The above fact, for some discrete $V$ in the Lie algebra of $N^{\prime}$, is then fairly immediate from the proof that lattices in nilpotent Lie groups are arithmetic. One needs to be slightly careful with the arguments to see that one can then realize $V$ as a (non-discrete) subgroup of $\mathfrak{n}$ with the desired properties. One can use the argument in this paper to give a slightly more direct proof of the arithmeticity of lattices in nilpotent Lie groups.

Our argument uses nilpotency in an essential way. (The Baker-Campbell-Hausdorff formula is finite!) There is some speculation among experts that all symmetric multiplicative subgroups of an arbitrary group $G$ may arise from subsets of nilpotent groups such as those arising in our theorem; see e.g. [5, Chapter 3.2] and the references there.

\section{Proofs}

Let $N$ be a simply connected nilpotent Lie group of nilpotency $n$. It is known that exp, the exponential map, is a diffeomorphism and we let $\log : N \rightarrow \mathfrak{n}$ be its inverse. For any $x, y \in N, q \in \mathbb{Q}$, we write $x^{q}$ to mean $\exp (q \log (x))$, and $c(x, y)$ to 
mean their commutator $x y x^{-1} y^{-1}$. For any positive integers $L$ and $j \geq 1$, we write $\Omega_{j}^{L}$ to mean all the possible maps from $\{1,2,3, \ldots, j\}$ to $\{1,2,3, \ldots, L\}$. If $\alpha \in \Omega_{j}^{L}$, we define $|\alpha|:=j$. Whenever we write $\prod_{\iota \in \Omega_{j}^{L}} x_{\iota}$, we mean multiplying from left to right with respect to a pre-fixed linear order on $\Omega_{j}^{L}$.

Recall the Baker-Campbell-Hausdorff formula, which says that for any $x, y \in N$,

$$
\log (x)+\log (y)=\log (x y)+\sum_{j=2}^{n} \sum_{\alpha \in \Omega_{j}^{2}} t_{\alpha} h_{\alpha},
$$

where each $t_{\alpha}$ is a rational number and $h_{\alpha}=\left(a d X_{1}\right) \circ\left(a d X_{2}\right) \circ \cdots \circ\left(\operatorname{ad} X_{|\alpha|-1}\right) X_{|\alpha|}$, with $X_{j}$ equal to $\log (x)$ if $\alpha(j)=1$ and equal to $\log (y)$ if $\alpha(j)=2$.

Lemma 2.1. Take $x_{1}, \ldots, x_{L} \in N$, and let $X_{i}=\log \left(x_{i}\right)$. For any $\alpha \in \Omega_{j}^{L}$, we write $h_{\alpha}$ to mean $\left(a d X_{\alpha(1)}\right) \circ \cdots \circ\left(a d X_{\alpha(j-1)}\right) X_{\alpha(j)}, H_{\alpha}$ to mean $c\left(x_{\alpha(1)}, c\left(x_{\alpha(2)}, \ldots\right.\right.$, $\left.\left.c\left(x_{\alpha(j-1)}, x_{\alpha(j)}\right)\right)\right)$. Then

(1)

$$
\log \left(x_{1} x_{2} \cdots x_{L}\right)=\sum_{i=1}^{L} X_{i}+\sum_{j=2}^{n} \sum_{\alpha \in \Omega_{j}^{L}} c_{\alpha} h_{\alpha}, c_{\alpha} \in \mathbb{Q} .
$$

(2) For any $j$ and $\alpha \in \Omega_{j}^{L}$ (remember $L$ is fixed),

$$
\log \left(H_{\alpha}\right)=h_{\alpha}+\sum_{\ell=j+1}^{n} \sum_{\beta \in \Omega_{\ell}^{L}} s_{\beta} h_{\beta}, s_{\beta} \in \mathbb{Q} .
$$

(3) For any $1 \leq J \leq n$, there are rational numbers $\beta_{2}, \beta_{3}, \ldots, \beta_{n}$ depending only on $J$ and $L$ so that

$$
\sum_{i=1}^{L} X_{i}=\log \left(x_{1} x_{2} \cdots x_{L}\right)+\sum_{i=2}^{J} \beta_{i} \log \left(\tilde{M}_{i}\right)+\sum_{i=J+1}^{n} \sum_{\alpha \in \Omega_{i}^{L}} c_{\alpha}^{\prime \prime} h_{\alpha},
$$

where $\tilde{M}_{i}=\prod_{\alpha \in \Omega_{i}^{L}}\left(H_{\alpha}\right)^{m_{\alpha}}$ for integers $m_{\alpha}$.

(4) For any $1 \leq j \leq n$, there are integers $c_{1}, c_{2}, \ldots, c_{n}$ (depending only on $j$ and $L)$ such that whenever an integer $T$ is divisible by each $c_{j}$,

$$
\sum_{\iota} T X_{\iota}=\log \left(\prod_{i=1}^{L} x_{i}^{T} \prod_{\ell=2}^{j} \prod_{\alpha \in \Omega_{\ell}^{L}} \tilde{H}_{\alpha}\right)+\sum_{\ell=j+1}^{n} \sum_{\alpha \in \Omega_{\ell}^{L}} s_{\alpha}^{\prime} h_{\alpha}, s_{\alpha}^{\prime} \in \mathbb{Q},
$$

where each $\tilde{H}_{\alpha}=c\left(x_{\alpha(1)}^{m_{\alpha(1)}}, c\left(x_{\alpha(2)}^{m_{\alpha(2)}}, \ldots, c\left(x_{\alpha(j-1)}^{m_{\alpha(j-1)}}, x_{\alpha(j)}^{m_{\alpha(j)}}\right)\right)\right)$ for some integers $m^{\alpha(i)}$ (depending on $T$ ).

In particular, in the case of (4), we are interested in the case $j=n$. We have stated the result with the parameter $j$ so that it is easy to write down a proof which is an induction.

Proof. The proof of (11) is a repeated application of Baker-Campbell-Hausdorff. Suppose the claim is true up to $J$. Then

$$
\log \left(x_{1} x_{2} \cdots x_{J}\right)=\sum_{i=1}^{J} X_{i}+\sum_{\ell=2}^{n} \sum_{\alpha \in \Omega_{\ell}^{J}} c_{\alpha} h_{\alpha}
$$


Applying (1) to $x_{1} x_{2} \cdots x_{J}$ and $x_{J+1}$ gives us

$$
\log \left(x_{1} x_{2} \cdots x_{J} \cdot x_{J+1}\right)=\log \left(x_{1} x_{2} \cdots x_{J}\right)+\log \left(x_{J+1}\right)+\sum_{j=2}^{n} \sum_{\alpha \in \Omega_{j}^{2}} t_{\alpha} h_{\alpha},
$$

where each $h_{\alpha}=\left(a d X_{1}\right) \circ\left(a d X_{2}\right) \circ \cdots \circ\left(a d X_{|\alpha|-1}\right) X_{|\alpha|}$ with $X_{j}$ equal to either $\log \left(x_{J+1}\right)$ or $\log \left(x_{1} x_{2} \cdots x_{J}\right)$. Substituting the latter by the inductive hypothesis yields (11) for $J+1$.

We now prove (2) by induction on $|\alpha|$. The base step (when $|\alpha|=2$ ) is obtained by combining (11) with the following:

$$
\log \left(e^{a} e^{b} e^{-a}\right)=b+\sum_{j=1}^{n-1} \frac{1}{j !}(a d(a))^{j}(b) .
$$

Suppose now that (2) is true up to $J$ many arguments. Then,

$$
\begin{aligned}
\log \left(c(x_{1}, \underbrace{c\left(x_{2}, \ldots, c\left(x_{J-1}, c\left(x_{J}, x_{J+1}\right)\right)\right.}_{=})\right) & {\left[X_{1}, \log \left(c\left(x_{2}, \ldots, c\left(x_{J-1}, c\left(x_{J}, x_{J+1}\right)\right)\right)\right)\right] } \\
& +\sum_{\ell=2}^{n} \sum_{\alpha \in \Omega_{\ell}^{2}} t_{\alpha} h_{\alpha} \\
= & {\left[X_{1},\left[X_{2},\left[\cdots,\left[X_{J}, X_{J+1}\right]\right]\right]\right]+\sum_{\ell=J+2}^{n} \sum_{\alpha \in \Omega_{\ell}^{J+1}} s_{\alpha} h_{\alpha}, }
\end{aligned}
$$

where the first line comes from applying the base step to $x_{1}$ and the underlined expression, and the second line comes from applying the inductive assumption. (11).

Equation (3) can be proved similarly by inducting on $J$. The case $J=1$ is just

Suppose it is true for $J$. Then

$$
\sum_{i=1}^{L} X_{i}=\log \left(x_{1} x_{2} \cdots x_{L}\right)+\sum_{i=2}^{J} \beta_{i} \log \left(\tilde{M}_{i}\right)+\sum_{\alpha \in \Omega_{J+1}^{L}} t_{\alpha} h_{\alpha}+\sum_{i=J+2}^{n} \sum_{\alpha \in \Omega_{i}^{L}} c_{\alpha}^{\prime \prime} h_{\alpha} .
$$

Let $\beta_{J+1}$ be 1 over the smallest common multiple of the denominators of the $t_{\alpha}$ 's, where $\alpha$ ranges over $\Omega_{J+1}^{L}$. Then the second summand above can be replaced by

$$
\begin{aligned}
\sum_{\alpha \in \Omega_{J+1}^{L}} t_{\alpha} h_{\alpha} & =\beta_{J+1} \sum_{\alpha \in \Omega_{J+1}^{L}} m_{\alpha} h_{\alpha}, m_{\alpha} \in \mathbb{Z} \\
& =\beta_{J+1} \sum_{\alpha \in \Omega_{J+1}^{L}} \log \left(H_{\alpha}^{m_{\alpha}}\right)+\sum_{i=J+2}^{n} \sum_{\alpha \in \Omega_{i}^{L}} t_{\alpha}^{\prime} h_{\alpha} \\
& =\beta_{J+1} \log \left(\prod_{\alpha \in \Omega_{J+1}^{L}} H_{\alpha}^{m_{\alpha}}\right)+\sum_{j=J+2}^{n} \sum_{\alpha \in \Omega_{j}^{L}} t_{\alpha}^{\prime \prime} h_{\alpha},
\end{aligned}
$$

where the second line comes from applying (2) to each term in the sum on the right hand side of the first line, and the third line comes from applying (1) followed by (21) to the first sum on the second line. 
We now prove (4) by induction on $j$. The base step is just (11). Suppose it is true up to $J$. If we let $c_{J+1}$ be the lowest common multiple between $c_{J}$ and the denominators of the $s_{\alpha}^{\prime}$ 's with $|\alpha|=J+1$, then using (3) of the lemma, we have

$$
\sum_{i=1}^{L} c_{J+1} X_{i}=\log \left(\prod_{i=1}^{L} x_{i}^{c_{J+1}} \prod_{j=2}^{J} \prod_{\beta \in \Omega_{j}^{L}} \tilde{H}_{\beta}\right)+\sum_{\alpha \in \Omega_{J+1}^{L}} \tilde{h}_{\alpha}+\sum_{\ell=J+2}^{n} \sum_{\beta \in \Omega_{\ell}^{L}} s_{\beta}^{\prime} h_{\beta},
$$

where $\tilde{h}_{\alpha}=\operatorname{ad}\left(m_{\alpha(1)} X_{\alpha(1)}\right) \circ \operatorname{ad}\left(m_{\alpha(2)} X_{\alpha(2)}\right) \cdots a d\left(m_{\alpha(J)} X_{\alpha(J)}\right)\left(m_{\alpha(J+1)} X_{\alpha(J+1)}\right)$ for integer $m_{\alpha}$ 's.

Applying (22) to the second term on the right hand side, we have

$$
\sum_{i=1}^{L} c_{J+1} X_{i}=\log \left(\prod_{i=1}^{L} x_{i}^{c_{J+1}} \prod_{j=2}^{J} \prod_{\beta \in \Omega_{j}^{L}} \tilde{H}_{\beta}\right)+\sum_{\alpha \in \Omega_{J+1}^{L}} \log \left(\tilde{H}_{\alpha}\right)+\sum_{\ell=J+2}^{n} \sum_{\beta \in \Omega_{\ell}^{L}} s_{\beta}^{\prime \prime} h_{\beta} .
$$

On the right hand side, we can combine the first term and each summand in the second term one at a time by applying (11) and using (11), (2) to get the desired equality.

We proceed towards the proof of the main theorem, but we first need the following lemma.

Lemma 2.2. For all $n$ there exists an integer $m$ depending only on $n$ so that if $a, b \in G$ with $G$ a simply connected $n$-step nilpotent Lie group, then

$$
m(\log a+\log b)=\log \left(w_{m, n}(a, b)\right),
$$

with $w_{m, n}(a, b)$ a word in $a, b, a^{-1}, b^{-1}$ with length $l(m, n)$ depending only on $m, n$.

Proof. This is an immediate consequence of Lemma 2.1. part (4).

Now we are ready to prove the main theorem.

Proof. Our first goal is to show that

$$
\left|\log \left(A^{k_{1}}\right)+\log \left(A^{k_{2}}\right)+\cdots+\log \left(A^{k_{l}}\right)\right| \lesssim|\log (A)|,
$$

where the constants depend only on the step $n$, the maximum of the $\left|k_{j}\right|$ 's, $l$ and the multiplicative constant of $A$. This follows directly from Lemma 2.2. Applying that lemma repeatedly, we find that every element of $\log \left(A^{k_{1}}\right)+\log \left(A^{k_{2}}\right)+\cdots+\log \left(A^{k_{l}}\right)$ is contained in $\frac{1}{m} \log \left(A^{l(m, n)}\right)$. Because $A$ is an approximate multiplicative subgroup we have that

$$
\left|A^{l(m, n)}\right| \lesssim|A|,
$$

and we have proved the first part.

Next we must show that

$$
B_{j} \subset q_{1} \log \left(A^{k_{1}}\right)+q_{2} \log \left(A^{k_{2}}\right)+\cdots+q_{l} \log \left(A^{k_{l}}\right) .
$$

We prove this by induction. Clearly it is true for $B_{0}$. Let us suppose that it is true for $B_{j-1}$. Then applying Lemma 2.2 to the induction hypothesis repeatedly, we find $m$ and $k$ depending only on $j$ and $n$ so that for any $b \in B_{j-1}$ we have

$$
e^{m b} \in A^{k} \text {. }
$$

We recall again the identity

$$
\log \left(e^{a} e^{m b} e^{-a}\right)=m b+\sum_{j=1}^{n-1} \frac{1}{j !} a d(a)^{j} m b .
$$


For any $1 \leq s \leq n-1$ with $s$ an integer, this implies that

$$
\log \left(e^{s a} e^{m b} e^{-s a}\right)=m b+\sum_{j=1}^{n-1} \frac{s^{j}}{j !} a d(a)^{j} m b .
$$

Viewing this as a system of $n$ linear equations for the unknowns $a d(a)^{j} b$, we can solve by inverting the Vandermonde matrix and finding rationals $q_{1}, \ldots, q_{n}$ depending only on $n$ and $j$ so that if $a \in \log (A)$ and $b \in B_{j-1}$, then we have

$$
[a, b] \in q_{1} \log \left(A^{k+2}\right)+q_{2} \log \left(A^{k+4}\right)+\cdots+q_{n} \log \left(A^{k+2 n}\right) .
$$

Thus we are done.

\section{ACKNOWLEDGEMENTS}

The first author is partially supported by NSF grant no. 0643546 and a fellowship from the Radcliffe Institute for Advanced Studies. The second author is partially supported by NSF grant no. 0653763.

\section{REFERENCES}

[1] Malcev, A. I., On a class of homogeneous spaces. Amer. Math. Soc. Translation 1951 (1951). no. 39, 33 pp. MR0039734 (12:589e)

[2] Raghunathan, M. S., Discrete Subgroups of Lie Groups. Ergebnisse der Mathematik und ihrer Grenzgebiete, Band 68. Springer-Verlag, New York-Heidelberg, 1972. MR.0507234 $(58: 22394 \mathrm{a})$

[3] Tao, Terence; Vu, Van, Additive Combinatorics. Cambridge Studies in Advanced Mathematics, 105. Cambridge University Press, Cambridge, 2006. MR.2289012 (2008a:11002)

[4] Tao, Terence, Product set estimates for non-commutative groups, Combinatorica 28 (2008), no. 5, 547-594. MR 2501249

[5] Tao, Terence, Structure and Randomness: Pages from Year One of a Mathematical Blog, American Mathematical Society, Providence, RI, 2008. MR2459552

Department of Mathematics, Indiana University, Bloomington, Indiana 47405

E-mail address: fisherdm@indiana.edu

Department of Mathematics, Indiana University, Bloomington, Indiana 47405

E-mail address: nhkatz@indiana.edu

Department of Mathematics, Indiana University, Bloomington, Indiana 47405

E-mail address: kanamejun@gmail.com 